\title{
Defining the Clinical Syndrome of Lumbar Spinal Stenosis: A Recursive Specialist Survey Process
}

\author{
Danielle E. Sandella, BS, Andrew J. Haig, MD, Christy Tomkins-Lane, PhD, \\ Karen S.J. Yamakawa, MS
}

Background: Lumbar spinal stenosis has evolved from an anatomic concept to a poorly defined clinical syndrome. Rules for such a syndrome need to be informed by the experience and beliefs of expert clinicians. The level of certainty is seldom considered in defining criteria for a syndrome.

Objective: To design an innovative online recursive survey technique to seek out information that is valued by specialists and to measure the impact of this evidence on their strength of conviction regarding the diagnosis of spinal stenosis.

Design: Prospective online survey.

Setting: University-based project.

Participants: American physiatrists recruited by online postings and postcards.

Interventions: A recursive process presented a scenario that allowed clinicians to choose 1 of 10 clinical factors and then asked their level of certainty about diagnosis when that factor is true. Subsequent questions build on that assumption by adding other factors.

Main Outcome Measures: Certainty regarding the diagnosis of clinical lumbar spinal stenosis.

Results: Of a total of 97 participants, 80 completed 3 or more iterations. "Leg pain while walking" (66\%), "must sit down or bend" (66\%), and "flex forward while walking" (49\%) were the most commonly selected questions. "Normal foot pulses" (19\%), "back pain" (16\%), "leg pain" (15\%), "relief with rest" (14\%), and "sensory deficits" (12\%) were of intermediate value, whereas "problems with balance," "have fallen recently," and "the sacroiliac joint is not the main pain generator" were all chosen less than $5 \%$ of the time. Statistically significant $(P<.05)$ change in certainty ceased after 6 questions at $86.2 \%$ certainty.

Conclusions: A recursive approach to diagnostic certainty is valuable. Within 5 questions, clinicians become almost $90 \%$ certain that a person has clinical spinal stenosis. This question set provides one pragmatic clinical criterion for the syndrome of lumbar spinal stenosis.

PM R 2013;5:491-495

\section{INTRODUCTION}

Lumbar spinal stenosis is a common diagnosis in older adults; however, a precise definition of stenosis is not available [1-3]. Although narrowing of the spinal canal is considered the hallmark of stenosis, the diameter of the spinal canal varies so greatly among patients that no acceptable point of demarcation exists between asymptomatic and symptomatic persons [4]. In addition, a person with a "narrow" spinal canal according to a magnetic resonance imaging scan may present with no pain or other symptoms. In such a patient, although a small canal may be present, treatment is not indicated $[2,4]$.

Consensus as to the definition of spinal stenosis has not been reached among experts. The "gold standard" for diagnosis and treatment of stenosis does not exist because of variable signs and symptoms, physicians' history-taking and physical examination methods, and diagnostic tests. The use of electromyography can help formulate a diagnosis, but it is not a practical procedure for an initial clinic visit or during the early diagnostic phases. Furthermore, electromyography has limited sensitivity for identifying mild spinal stenosis

D.E.S. Department of Physical Medicine and Rehabilitation, The University of Michigan, Ann Arbor, Ml

Disclosure related to this publication: grant, $\mathrm{NIH}$

A.J.H. Department of Physical Medicine and Rehabilitation, The University of Michigan, 325 E Eisenhower, Ann Arbor, Ml 48108. Address correspondence to: A.J.H.; e-mail: andyhaig@umich.edu

Disclosure related to this publication: grant, $\mathrm{NIH}$

Disclosures outside this publication: board membership, no funds received, International Society for Physical and Rehabilitation Medicine, and International Rehabilitation Forum; consultancy, direct funds received, The Center for Healthcare Research and Transformation; expert testimony, both direct funds received and money to institution, occasional legal case; grants/grants pending, National Institutions of Health; grants/grants pending, direct funds received, The Center for Healthcare Research and Transformation, Community, and Emergency Department interventions for back pain; payment for lectures including service on speakers bureaus, miscellaneous academic institutions; payment for development of educational presentations, Jerudong Park Medical Center, Brunei, development of a national rehabilitation center

C.T.-L. Mount Royal University, Calgary, Alberta, Canada

Disclosure: nothing to disclose

K.S.J.Y. Department of Physical Medicine and Rehabilitation, The University of Michigan, Ann Arbor, Ml

Disclosure related to this publication: grant $\mathrm{NIH}$

Presented at American Academy of Physical Medicine and Rehabilitation, Atlanta, GA, November 15-18, 2012.

The project described was supported by Award Number R01HD059259 from the Eunice Kennedy Shriver National Institute of Child Health \& Human Development. The content is solely the responsibility of the authors and does not necessarily represent the official views of the Eunice Kennedy Shriver National Institute of Child Health \& Human Development or the National Institutes of Health.

Peer reviewers and all others who control content have no relevant financial relationships to disclose.

Submitted for publication July 8, 2012; accepted October 2, 2012. 
[5] and therefore, like magnetic resonance imaging, it cannot be considered a foolproof method for diagnosis.

Suri et al [6] defined stenosis as requiring both the presence of radiographic stenosis and clinical symptomatology. Konno et al [7] developed a patient self-report form to assist in the diagnosis of stenosis; however, this tool has many limitations. First, the diagnostic tool contains only 10 questions and focuses on the degree of the patient's numbness. Although the authors performed various statistical analyses when developing their tool, it is unclear whether there is truly an expert consensus that numbness is a main indicator of stenosis and whether it is specific to stenosis. In addition, the diagnostic tool does not address diagnoses that present with similar symptoms as stenosis, such as vascular disease, mechanical low back pain, or diabetic neuropathy. Although this tool may be useful in shortening the length of the history during the office visit, the physician will still need to examine and interview the patient to make a final diagnosis.

It is important to establish a set of core diagnostic criteria for spinal stenosis determined by patient history and physical examination and independent of other diagnostic tests. Suri et al [6] discussed the value of an accurate history and physical examination but did not define which items were the most useful in diagnosing lumbar spinal stenosis. In that study, certain items, when answered affirmatively by the patient, were shown to increase the likelihood of a diagnosis of spinal stenosis; however, the authors did not include the physicians' level of certainty of the diagnosis or the frequency with which physicians actually ask each question when taking a history, probably because such information has not been studied and therefore is unavailable in the literature.

Syndromes are sets of rules. In the past, the development of rules for syndromes largely has been conducted by expert panels. Although these panels often are well informed by previous literature and personal experience, it is presumptive to believe that such panels have a better perspective than the collective wisdom of hundreds or thousands of clinicians or that they represent common beliefs. Furthermore, such panels are by nature political, and academic experts may be more likely to defend their publicized beliefs than anonymous clinicians. Finally, especially early in the development of syndrome definitions, the diagnostic certainty obtained from using different rule sets is not evaluated.

The advent of sophisticated Web-based survey instruments provides a unique opportunity to transcend these issues. We have developed an online recursive survey methodology that permits anonymous community-based specialists to express the value that they place on certain clinical items, the logical order in which they consider them, and the level of certainty that is ascertained from the data that they have chosen.

'The main focus of the present study was to collect the history and physical examination information (in response to a set of predetermined questions) that specialist clinicians believe defines the clinical syndrome of lumbar spinal stenosis; to determine the relative weights and logical process behind these choices; and to measure the level of certainty that clinicians believe can be obtained through use of this syndrome definition.

\section{METHODS}

Through an ethical review board-approved process, a set of potentially relevant clinical questions was established, which was codified into an online survey promoted online to physical medicine and rehabilitation physicians practicing in the United States. Our analysis evaluated the choice of questions, the weight given to answers, and the physicians' overall certainty.

\section{Development of the Question Set}

An initial question set was developed through the following methodology. Initial brainstorming was performed during a meeting of clinicians and researchers involved with the Michigan Spinal Stenosis Study II study [8]. The group included a neurosurgeon, a vascular surgeon, an electromyographer, 5 physiatrists, and a radiographer. Clinicians' perceptions of the signs and symptoms of spinal stenosis were listed. The clinicians were then asked to rate the specificity and sensitivity of each sign or symptom by using a visual analog scale.

After the initial brainstorming, a formal survey was developed to determine which symptoms were most important to the clinicians when diagnosing spinal stenosis. Thirty signs and symptoms were included, and clinicians involved with the Michigan Spinal Stenosis Study II, including those present at the previous meeting and others who were not present, completed the survey. The top 15 signs and symptoms as determined by the initial survey and the most important symptoms that appeared in the interim in an article by Suri et al [6] were used in developing a survey to further define clinical criteria for spinal stenosis.

\section{The Online Survey}

The survey was constructed through an online portal of the University of Michigan with the use of Qualtrics survey software (Qualtrics Labs, Inc, Provo, UT). Responders were provided with minimal information about a patient who presents in their clinic. Specifically, "A patient, over 65 years old, comes into your office with symptoms they attribute to the low back or leg." The responders were then asked, "You are interested in finding out if they have the clinical syndrome of lumbar spinal stenosis. What is the first question you would ask?" On the basis of the selected question, the respondent is informed of the patient's answer and then asked to rate, on the basis of all known information about the patient and the patient's symptoms, how certain the re- 
sponder was that the patient had spinal stenosis on a sliding bar akin to a visual analog scale, anchored by 0 , which represented "not at all certain," and 100, which represented "completely certain." This process was repeated up to 10 times or until the responder quit. Plausible but likely irrelevant questions such as "presence of thyroid dysfunction" and "headaches," as seen in Figure 1, were used to ensure true participation versus random-choice selection.

The survey was distributed to physiatrists, spine surgeons, and others through e-mail messages to colleagues and encouragement of these colleagues to forward it to others. The survey also was posted on the American Academy of Physical Medicine and Rehabilitation's Pain Forum; however, we did not have access to online groups representing non-Americans, surgeons, or other groups. Although a handful of nonphysiatrists and non-Americans responded, as noted in the Discussion, this limitation in distribution led to a post-hoc decision to describe the results from only the American physiatrist population in the current article.

Statistical analysis was performed by downloading data from the Web site software to a PASW Version 17 (SPSS, Inc, Chicago, IL) spreadsheet. Most statistics were descriptive; however, a paired-sample $t$-test was used to determine significant changes in certainty over time, with $P<.05$ considered significant.

\section{RESULTS}

One hundred twenty-nine physicians, primarily physiatrists, participated. Participants included physicians from 12 countries, including the United States, Canada, Brazil, and others. For the purposes of this study, data analysis was restricted to the respondents who indicated that they were physiatrists practicing in the United States. The average number of years in practice was 9.8 and ranged from 1 to 35 years. Fifty-seven percent of the participants worked in private practice, whereas 29\% worked at a hospital or other large-scale practice. All participants indicated they knew the meaning of the

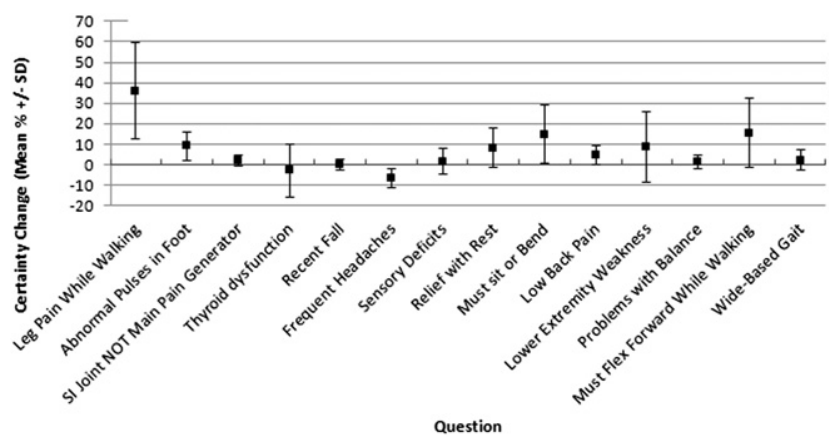

Figure 1. Influence of questions on certainty: the average percentage change created by each question. SI = sacroiliac.

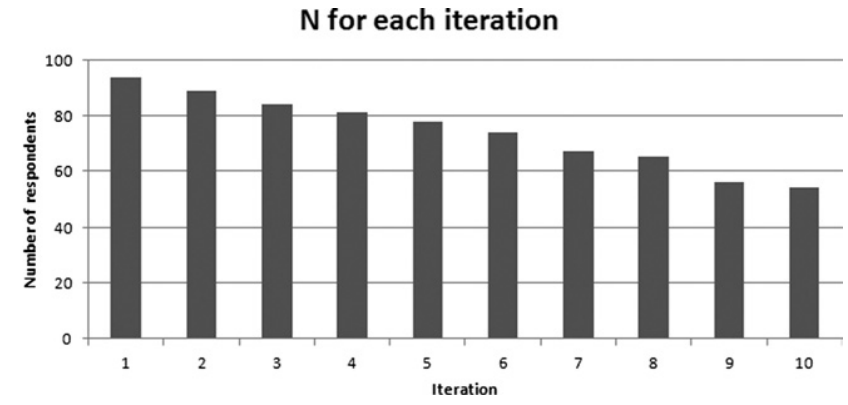

Figure 2. The number of respondents who completed " $n$ " iterations of the survey question.

term "spinal stenosis" and were willing to participate. Of the original 129 participants, 97 were kept for analysis. Fiftyfour participants completed the entire survey, whereas 30 completed at least 3 iterations but did not complete the process, and the remaining 13 completed fewer than 3 iterations (Figure 2).

To determine the questions that were most important to physicians in diagnosing spinal stenosis, multiple analyses were performed with regard to which questions were asked when and how certain the physician felt of his or her diagnosis after each question. Figure 3 shows the frequency of question selection after each iteration. Leg pain while walking, the need to sit down or bend forward to relieve pain, and flexing forward while walking were the most commonly asked questions, with 66, 66, and 49 participants selecting these questions in the first 3 iterations, respectively.

We determined the impact of each question on physician certainty by calculating the change in certainty before and after the question was asked, regardless of question order. Figure 1 depicts the means and SD of these changes in certainty and shows that the same 3 questions that were asked most often also had the greatest positive impact on certainty.

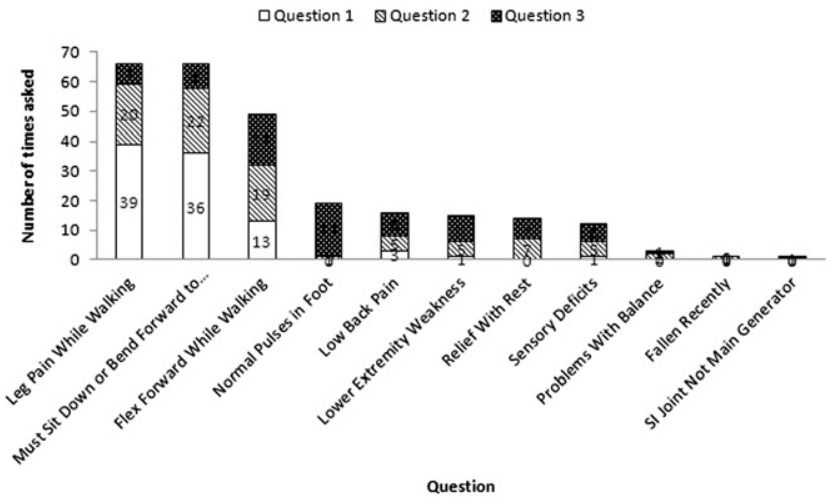

Figure 3. Value in decision making. The distribution of the clinician's choice of information in the first 3 questions. SI = sacroiliac. 


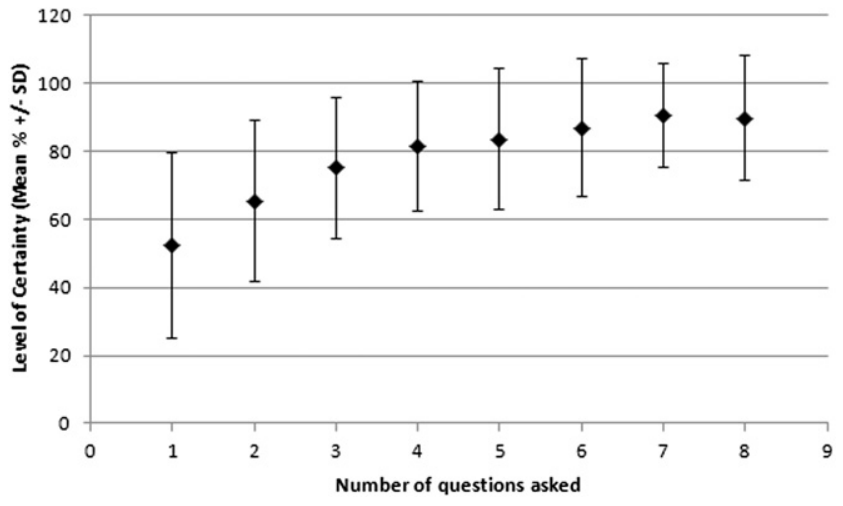

Figure 4. Clinician confidence: diagnostic certainty as determined by the number of questions asked.

Finally, the physicians' certainty of diagnosis based on the total number of questions asked (Figure 4; Table 1) was determined to establish whether a maximal level of certainty is achieved after a particular number of questions. A significant difference in certainty levels through 6 questions was found; after the sixth iteration, additional questions did not result in significant increases in certainty.

\section{DISCUSSION}

In this study we used an innovative process to establish clinical criteria and certainty about the diagnosis of lumbar spinal stenosis by using the expertise of a broad-based group of clinicians. The strengths and weaknesses of this methodology and the implications regarding spine care need to be explored.

The recursive design of this study is unlike any of which we are aware in spine research. The process mimics clinical practice in which factors are weighted against each other, and sometimes sequentially, not in isolation. This approach largely bypasses biases among the typical small groups that form academic consensus. However, not all biases can be avoided. In this case, development of the questions by a broad-based specialist panel provides some face validity. In addition, the list of questions used and their value provides transparency. Within this particular study of lumbar stenosis, American physiatrists who care to take time to answer this survey may not represent non-Americans, nonphysiatrists, or those who are too busy or otherwise unable to respond. In the end, this transparent process allows critique and research on the reproducibility of the findings.

A number of important concepts are measured in this study: (1) Which factors are most important in the diagnosis? (2) How many questions are needed to gain reasonable certainty? (3) How certain can clinicians be after asking their questions?

This study was designed to evaluate factors that can be obtained during a routine clinical encounter. The order of questions, as seen in Figure 3, reflects some value, as does influence on decision making, illustrated in Figure 1. The first-chosen and highest-rated questions are compatible with findings of Suri et al [6], who found that the most important findings on history and physical examination were "absence of pain while seated" and "improvement of symptoms when bending forward." Other frequently chosen questions reflect common wisdom about clinical spinal stenosis. Less frequently chosen questions are not to be considered clinically useless, and indeed questions not even asked (eg, "Do you have a familial neuropathy?") have obvious value for a small percentage of patients. The questions are useful in defining a common consensus for research and policy.

This study also brought to light how many questions are needed to gain a reasonable amount of certainty of diagnosis. This asymptote is not at $100 \%$ perfection but at somewhere near $85 \%$. A recent study of spine surgeons who performed a comprehensive history and physical examination found that the surgeons also reached a level certainty for the diagnosis for lumbar stenosis at $7.81 \pm 1.91 \mathrm{~cm}$ on a $10-\mathrm{cm}$ visual analog scale, quite similar to the $80 \%$ noted here (K. Yamakawa et al, unpublished data, 2012). However, their forced-choice diagnosis sometimes related to an analog certainty of less than $50 \%$, and the surgeons even contradicted each other in some obvious cases (A. J. Haig et al, unpublished data, 2012).

The concept of certainty often is skirted in evidence-based medicine. Further study may find that diverse factors affect the level of a physician's certainty, ranging from his or her personality to competence to the consequences of the decision. Still, the questions in this study, leading to greater than $80 \%$ certainty, can be used by researchers who want to describe the level of certainty that a person has what is recognized by others as clinical spinal stenosis. This situation

Table 1. Clinician certainty about diagnosis as determined by the number of questions asked

\begin{tabular}{ccc}
\hline No. Questions Asked & Mean Certainty & Significance (P) \\
\hline 1 & 52.29 & \\
2 & 65.43 & $<.001^{*}$ \\
2 & 65.47 & $<.001^{*}$ \\
3 & 75.08 & \\
3 & 74.35 & .133 \\
4 & 81.26 & \\
4 & 81.04 & $.028^{*}$ \\
5 & 83.52 & $.009^{*}$ \\
5 & 83.01 & .88 \\
6 & 86.91 & \\
6 & 86.18 & .542 \\
7 & 90.36 & .119 \\
7 & 89.4 & \\
8 & 89.66 & \\
8 & 88.58 & \\
9 & 86.64 & \\
\hline
\end{tabular}

*Significance $P<.05$ based on paired sample $t$-test. 
is an improvement over simply stating that subjects "met criteria."

A recent survey of surgeons by Schizas and Kulik [8] provides a platform to demonstrate the utility of this study's findings. In their study, surgeons were asked essentially one question: "Assuming the MR images correspond to $20 \mathrm{pa}-$ tients who have severe neurological claudication symptoms and who are fit for surgery, would you consider decompressing their lumbar spine given the degree of stenosis shown on those 20 images?" The authors found that 20\% would have operated on persons with a certain "type Al" copious spinal canal and 80\%-100\% would operate on tight canals termed type B, C, and D. Szhizas' study might have been greatly improved by presentation of a case scenario that has been proven to result in, for instance, $80 \%$ certainty regarding the clinical diagnosis rather than "severe neurogenic claudication."

The results of the current study are not the final word on a clinical definition of the syndrome of clinical lumbar stenosis. The acceptance of a definition of a syndrome may be informed by science, but in the end the process of accepting a definition is social and political. In the end, it, as useful as a "concrete" diagnostic criteria for stenosis can be, optimal care requires recursive interaction between diagnostic certainty, risks and benefits of treatments, and the needs and goals of the patient $[9,10]$. An approach that simply anoints a single diagnostic criterion does not serve well.

\section{CONCLUSION}

This study found that practicing physical medicine and rehabilitation clinicians approximate a consensus on the history and physical examination findings that diagnose clinical lumbar spinal stenosis. Their diagnostic certainty reached an asymptotic level of $87 \%$ after 6 questions. These findings can inform the establishment of an accepted clinical syndrome of lumbar spinal stenosis.
A consensus-based set of criteria for diagnosing lumbar spinal stenosis is important to the proper management of patients with this complicated disease. Such criteria can be established by medical policy makers informed by studies similar to this one. The result of potential future studies will be a better understanding and more consistent, appropriate treatment of patients with spinal stenosis.

\section{REFERENCES}

1. Goh K, Khalifa W, Anslow P, Cadoux-Hudson T, Donaghy M. The clinical syndrome associated with lumbar spinal stenosis. Eur Neurol 2004;52:242-249.

2. Haig AJ, Tomkins C. Diagnosis and management of lumbar spinal stenosis. JAMA 2010;303:71-72

3. Truumees E. Spinal stenosis: Pathophysiology, clinical and radiologic classification. Instr Course Lect 2005:54:287-302.

4. Haig AJ. Correlation between disability and MRI findings in lumbar spinal stenosis. Acta Orthop 2011;82:637; author reply 637-638.

5. Chiodo A, Haig AJ, Yamakawa KS, Quint D, Tong H, Choksi VR. Needle EMG has a lower false positive rate than MRI in asymptomatic older adults being evaluated for lumbar spinal stenosis. Clin Neurophysiol 2007;118:751-756

6. Suri P, Rainville J, Kalichman L, Katz JN. Does this older adult with lower extremity pain have the clinical syndrome of lumbar spinal stenosis? JAMA 2010;304:2628-2636.

7. Konno S, Kikuchi S, Tanaka Y, et al. A diagnostic support tool for lumbar spinal stenosis: A self-administered, self-reported history questionnaire. BMC Musculoskelet Disord 2007:8:102.

8. Schizas C, Kulik G. Decision-making in lumbar spinal stenosis: A survey on the influence of the morphology of the dural sac. J Bone Joint Surg Br 2012;94B:98-101.

9. Reuben $\mathrm{DB}$, Tinetti ME. Goal-oriented patient care-an alternative health outcomes paradigm. N Engl J Med 2012;366:777-779.

10. Barry MJ, Edgman-Levitan S. Shared decision making-pinnacle of patient-centered care. N Engl J Med 2012;366:780-781.

This CME activity is designated for 1.0 AMA PRA Category 1 Credit ${ }^{\mathrm{TM}}$ and can be completed online at me.aapmr.org. Log on to www.me.aapmr.org, go to Lifelong Learning (CME) and select Journal-based CME from the drop down menu. This activity is FREE to AAPMER members and $\$ 25$ for non-members.

\section{CME Question}

The most common clinical finding associated with lumbar spinal stenosis, as determined by a recursive survey of physicians, was:

a. sensory deficits

b. relief of pain with rest

c. leg pain while walking

d. normal foot pulses

Answer online at me.aapmr.org 